\title{
Protecting Axonal Degeneration by Increasing Nicotinamide Adenine Dinucleotide Levels in Experimental Autoimmune Encephalomyelitis Models
}

\author{
Shinjiro Kaneko, ${ }^{1}$ Jing Wang, ${ }^{1}$ Marie Kaneko, ${ }^{1}$ Glenn Yiu, ${ }^{1}$ Joanna M. Hurrell, ${ }^{1}$ Tanuja Chitnis, ${ }^{2}$ Samia J. Khoury, ${ }^{2}$ and \\ Zhigang $\mathrm{He}^{1}$ \\ ${ }^{1}$ Division of Neuroscience, Children's Hospital Boston, Harvard Medical School, and 2Brigham and Women's Hospital, Harvard Medical School, Boston, \\ Massachusetts 02115
}

\begin{abstract}
Axonal damage is a major morphological alteration in the CNS of patients with multiple sclerosis (MS) and its animal model, experimental autoimmune encephalomyelitis (EAE). However, the underlying mechanism for the axonal damage associated with MS/EAE and its contribution to the clinical symptoms remain unclear. The expression of a fusion protein, named "Wallerian degeneration slow" $\left(\right.$ Wld $\left.{ }^{\mathrm{s}}\right)$, can protect axons from degeneration, likely through a $\beta$-nicotinamide adenine dinucleotide (NAD)-dependent mechanism. In this study, we find that, when induced with EAE, Wld $d^{5}$ mice showed a modest attenuation of behavioral deficits and axon loss, suggesting that EAE-associated axon damage may occur by a mechanism similar to Wallerian degeneration. Furthermore, nicotinamide (NAm), an NAD biosynthesis precursor, profoundly prevents the degeneration of demyelinated axons and improves the behavioral deficits in EAE models. Finally, we demonstrate that delayed NAm treatment is also beneficial to EAE models, pointing to the therapeutic potential of NAm as a protective agent for EAE and perhaps MS patients.
\end{abstract}

Key words: axon degeneration; EAE; NAD; Wallerian degeneration; nicotinamide; Wld $^{\text {s }}$

\section{Introduction}

Multiple sclerosis (MS) is a chronic neurological disorder characterized by focal lesions with inflammation, demyelination, and neurodegeneration in the CNS. Although most patients begin with a relapsing-remitting course, the majority eventually will enter a chronic progressive phase with increasing clinical disabilities (Steinman, 2001; Bjartmar et al., 2003). Available treatments are primarily designed for antiinflammation, and are only effective during the relapsing-remitting phase. In addition, some of these agents such as Tysabri, a humanized monoclonal antibody to $\alpha 4$ integrin, may have severe side effects possibly resulting from immunosuppression (Adelman et al., 2005). Thus, developing novel therapeutic strategies, in particular those to prevent long-term neurological dysfunction, has been a pressing issue for MS patients.

Although it was formerly assumed that axons are spared from the destructive process, a variety of histological analyses has demonstrated that axonal damage occurs at multiple stages of MS and that axonal loss is progressive along the course of the disease (Trapp et al., 1998; Onuki et al., 2001). Importantly, evidence

Received May 18, 2006; revised Aug. 3, 2006; accepted Aug. 10, 2006

This work was supported by grants from the National Multiple Sclerosis Society and National Institute of Neurological Disorders and Stroke. Z.H. is a McKnight scholar. We thank Dr. David Rowitch for reading this manuscript and Drs. Ken-ichiro Kuwako, Masaaki Kitada, Thomas Diefenbach, Maria Ericsson, Lauren Friedman, and Jean Brennan for technical advice.

Correspondence should be addressed to Zhigang He, Division of Neuroscience, Children's Hospital Boston, Enders \#379, 320 Longwood Avenue, Boston, MA 02115. E-mail: zhigang.he@childrens.harvard.edu. D01:10.1523/JNEUROSCI.2116-06.2006

Copyright $\odot 2006$ Society for Neuroscience $\quad$ 0270-6474/06/269794-11\$15.00/0 points to a close correlation between the extent of axon damage and the permanent neurological dysfunction associated with the disease. However, the cellular and molecular mechanisms of MSassociated axon damage and the precise contribution of axon damage to MS remains mostly unclear. Importantly, an efficient strategy for axonal protection for MS patients is still lacking.

In principle, axon damage in MS can result from local immune attacks and accompanying demyelination, often occurring at multiple sites along different white matter tracts in the CNS of MS patients and experimental autoimmune encephalomyelitis (EAE) animal models. In acute MS plaques, although transected axons have been seen, many axons that are damaged by inflammation and demyelination remain physically connected (Matthews et al., 1998). If unprotected, such local damage may lead to transection observable as ovoid-like axons (Ferguson et al., 1997; Trapp et al., 1998), and culminate in the degeneration of the distal axonal segments and a reduction in axonal density. Thus, it is conceivable that protecting these vulnerable axons from degeneration might promote recovery from the disease course.

To some extent, the degenerative process resulting from local axonal damage in MS/EAE is reminiscent of "Wallerian degeneration," a self-destructive process observed at the distal portion of a transected axon after injury. Significant progress has recently been made in understanding the mechanisms of Wallerian degeneration, primarily from analyzing the mechanisms that account for the slow Wallerian degeneration phenotype of $W l d^{s}$ mice (Raff et al., 2002; Coleman, 2005; Luo and O'Leary, 2005). Genetic studies have attributed this phenotype to the overexpres- 
A

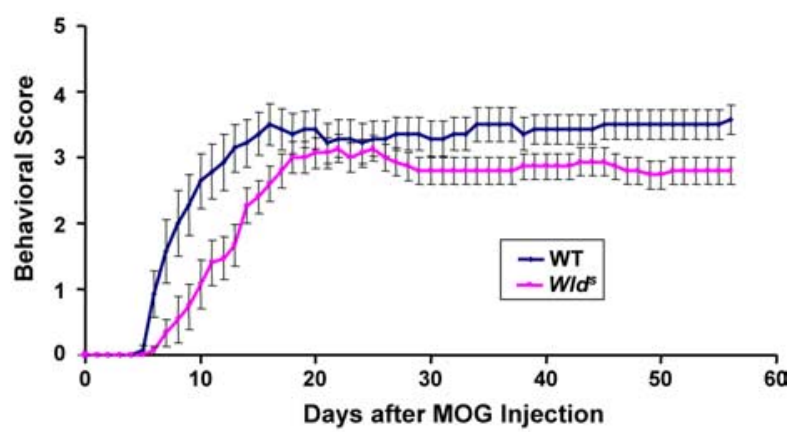

B

\begin{tabular}{|l|c|c|}
\hline & WT & W/d \\
\hline Incidence & $14 / 14$ & $15 / 15$ \\
\hline Death from Severe Disease & 3 & 1 \\
\hline Onset Day & $7.5 \pm 0.6$ & $* 10.1 \pm 0.7$ \\
\hline
\end{tabular}

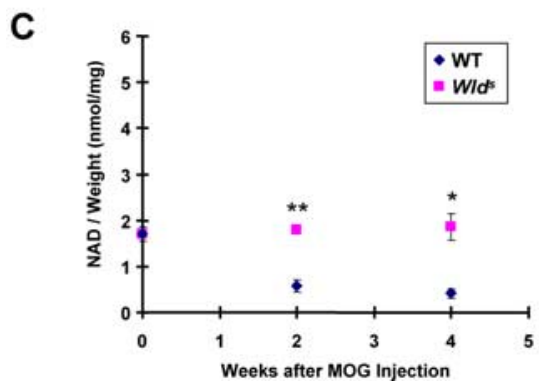

Figure 1. WId ${ }^{5}$ modestly delayed the onset and attenuated the behavioral deficits of EAE. $A$, Behavioral scores (mean $\pm \mathrm{SEM}$ ) of EAE in $\mathrm{C} 57 \mathrm{BL} / 6$ or WId $\mathrm{d}^{5}$ mice. Differences between these groups were significant as determined by two-tailed Student's $t$ test, $p<0.05$ from $6 \mathrm{~d}$ p.i. $\boldsymbol{B}$, Clinical features of EAE in C57BL/6 or WId $d^{5}$ mice. The onset was significantly delayed in WId $d^{5}$ mice when compared with the wild-type group $\left({ }^{*} p<0.01\right.$; Student's $t$ test). C, NAD levels (presented as mean \pm SEM) of the cervical spinal cords of EAE animals as analyzed by HPLC. In C57BL/ 6 mice, NAD levels at 2 or 4 weeks p.i. were significantly decreased compared with uninduced controls ( $p=0.0012, p=0.0004$ at 2 and 4 weeks p.i., respectively). However, the NAD levels in WId ${ }^{5}$ mice were preserved compared with those from wild-type EAE mice ${ }^{* *} p<$ $0.001,{ }^{*} p<0.01$ at 2 and 4 weeks p.i., respectively; Student's $t$ test).

sion of a fusion protein (Wld ${ }^{s}$ ) that consists of the full-length nicotinamide mononucleotide adenylyltransferase (Nmnat1), an enzyme required for nicotinamide adenine dinucleotide (NAD) biosynthesis, and a short region of a ubiquitin assembly protein, UFD2 (ubiquitin fusion degradation protein-2). In cultured neurons, both inhibition of the ubiquitin-proteosome system and overexpression of Wld ${ }^{s}$ or full-length Nmnat 1 can slow down Wallerian degeneration (Zhai et al., 2003; Araki et al., 2004; Wang et al., 2005). In additional support of the critical role of NAD in the protective effects of these NAD-synthesizing enzymes, we found that NAD levels decrease in degenerating axons and that preventing this axonal NAD decline by either overexpressing Wld ${ }^{\mathrm{s}}$ or Nmnatl, or exogenously supplying NAD or the NAD precursor nicotinamide (NAm), can efficiently protect axons against degeneration (Wang et al., 2005). In addition to mechanical injury, the $W l d^{s}$ allele has also been shown to protect against axonal degeneration in models such as the pmn motor neuron disease, P0 deficient mice, and SOD $1{ }^{\text {G93A }}$ ALS mice (for review, see Coleman, 2005).

Given the similarities between the pathology of MS-associated axon damage and Wallerian degeneration, we asked whether these processes share similar molecular mechanisms and whether treatment to prevent Wallerian degeneration could be exploited to develop protective strategies for MS and its animal models. In this study, we have used a combination of genetic and pharmacologic approaches to address these questions. We found a modest alleviation of behavioral deficits and axonal loss in Wld mice, suggesting that axon degeneration in the EAE model may be mechanistically similar to Wallerian degeneration. Importantly, we demonstrate that administering NAm can prevent the decline of neuronal NAD and result in profound neuroprotection in EAE model animals, providing a novel therapeutic possibility for MS patients.

\section{Materials and Methods}

Induction and behavioral assessment of EAE. C57BL/6 mice and Wld ${ }^{\mathrm{s}}$ mice with the same background (Harlan, Indianapolis, IN) and (PL/J $\times$ $\mathrm{SJL} / \mathrm{J}) \mathrm{F}_{1}$ mice (The Jackson Laboratory, Bar Harbor, ME) were used in this study. The myelin oligodendrocyte glycoprotein (MOG)-induced EAE model was generated following the procedure described by Kalyvas and David (2004). We scored the behavioral deficits daily for all of the mice in a double-blind manner, following a 5-point behavioral scoring scale as described previously (Kalyvas and David, 2004) (grade 1, flaccid tail; grade 2, mild hindlimb weakness; grade 3, severe hindlimb weakness; grade 4 , hindlimb paralysis; grade 5 , hindlimb paralysis and forelimb weakness or moribund). We determined onset as the day when a mouse was scored as grade 1 for the first time, except for the mice that remained at grade 0 until 8 weeks postimmunization (p.i.), whose onset day was counted as $57 \mathrm{~d}$ p.i. The proteolipid protein (PLP) 139-151 peptideinduced EAE model in $(\mathrm{PL} / \mathrm{J} \times \mathrm{SJL} / \mathrm{J}) \mathrm{F}_{1}$ mice was generated following the procedure described by Zhu et al. (2006).

For NAm treatment, we subcutaneously administered NAm (Sigma, St. Louis, MO) or PBS (as a control) daily from the day of inoculation. For the delayed treatment group, we injected PBS until $9 \mathrm{~d}$ p.i., and then began injecting NAm $(500 \mathrm{mg} / \mathrm{kg})$ from $10 \mathrm{~d}$ p.i.

Histochemistry and quantification. All animals for the initial groups used to examine behavioral deficits (11-16 animals per group) were terminated and used for histological examinations at 8 weeks p.i. Additional groups of animals ( $n=8$ per group) were subjected to EAE but terminated 2 weeks p.i. After perfusion and dissection, serial transverse sections $(15 \mu \mathrm{m})$ were cut from the cervical and one-half of the lumbar spinal cord, and the other one-half of the lumbar spinal cords was used for sagittal sections. Sections were used for Hoechst 33258 staining and immunostaining with antibodies against myelin basic protein (MBP), and neurofilament (NF)200. The areas of infiltration and demyelination were quantified by using SPOT software (Diagnostic Instruments, Sterling Heights, MI). Areas of infiltration $\left(10^{3} \mu \mathrm{m}^{2} /\right.$ section $)$ or demyelination $\left(10^{3} \mu \mathrm{m}^{2} /\right.$ section $)$ were determined as the measured Hoechst + or $\mathrm{MBP}-$ areas averaged from 10 transverse sections per animal for each group. The numbers of NF+ and MBP- axons in the demyelinated areas were also counted by using high-power microscopy from all of the stained transverse sections for statistical analysis. At $400 \times$ magnification, the axonal fibers are readily distinguishable from other possible artifacts such as pial/meningial structures, which were excluded from our quantifications. For CD4 and CD8 staining, six sagittal sections, taken from every 10 lumbar spinal cord sections of each animal in each group at 2 weeks p.i., were stained, and the numbers of positive cells costained with Hoechst 33258 per section were counted by using high-powered microscopy and compared statistically.

Immunostaining was performed following standard protocols. Primary antibodies used included rabbit polyclonal anti-NF200 (Serotec, Indianapolis, IN; 1:250), rat monoclonal anti-MBP (Abcam, Cambridge, MA; 1:50), and rat monoclonal anti-CD4 and anti-CD8 (BD Pharmingen, San Diego, CA; 1:100). For terminal deoxynucleotidyl transferasemediated biotinylated UTP nick end labeling (TUNEL), we used ApopTag Red In Situ (Chemicon, Temecula, CA).

Sample preparation and reverse-phase HPLC analysis. Sample preparation and HPLC analysis were performed as described previously (Wang et al., 2005) with minor modification. In brief, after perfusion with PBS, the cervical spinal cord was dissected from the mice on ice and the samples were processed following the method described by Wang et al. 
(2005). We harvested samples from three to four animals per group at each time point.

Coculture of cortical neurons and microglia. Embryonic day 18 (E18) rat cortical neurons were plated at $1 \times 10^{5}$ cells/well on poly-Dlysine-coated 24-well plates in Neurobasal medium (Invitrogen, San Diego, CA) containing $2 \%$ B-27 supplement and $0.6 \mathrm{~mm}$ L-glutamine and incubated in humidified air containing 5\% $\mathrm{CO}_{2}$. Microglia cell line BV2 (American Type Culture Collection, Manassas, VA) were cultured in RPMI 1640 (Invitrogen) supplemented with $10 \%$ heat-inactivated FBS and $1 \%$ penicillin/streptomycin and incubated in humidified air containing $5 \% \mathrm{CO}_{2}$ until confluent. In the treatment groups, NAm (25 $\mathrm{mm}$ ) was added to the neuronal culture $24 \mathrm{~h}$ after the plating. After another $24 \mathrm{~h}$, microglia $\left(1 \times 10^{5}\right.$ cells/well $)$ were seeded with primary neuronal cultures (at this point, NAm was removed from the medium in the "pretreatment only" group) and stimulated using $1 \mu \mathrm{g} / \mathrm{ml}$ lipopolysaccharide (LPS) for $60 \mathrm{~h}$. Cells were fixed with $4 \%$ paraformaldehyde for 20 min and stained with rabbit Tuj1 antibody (Covance, Princeton, NJ; 1:500) together with Hoechst 33258. Numbers of Tuj1-positive cells were quantified in triplicate in three independent experiments.

\section{Results}

Modest reduction of neurological disability of EAE in $W l d^{s}$ mice

To explore possible mechanisms for axon damage in MS/EAE, we first examined whether the $W l d^{s}$ gene affects the behavioral deficits associated with a chronic form of EAE. Thus, we followed a standard procedure to induce EAE by immunizing $W l d^{s}$ mice (C57BL/6 background) and control C57BL/6 mice with a MOG peptide (amino acids 35-55). Consistent with previous reports (Kalyvas and David, 2004; Aktas et al., 2005), the control mice subjected to this procedure developed a typical course of neurological disability that became obvious $\sim 10 \mathrm{~d}$ p.i. and persisted for at least 8 weeks (Fig. $1 A$ ). However, when subjected to the same procedure, $W l d^{s}$ mice showed a modest but statistically significant delay in the onset of behavioral deficits (Fig. $1 A, B$ ), as well as an attenuation of symptoms over the course of EAE development (Fig. 1A,B).
A
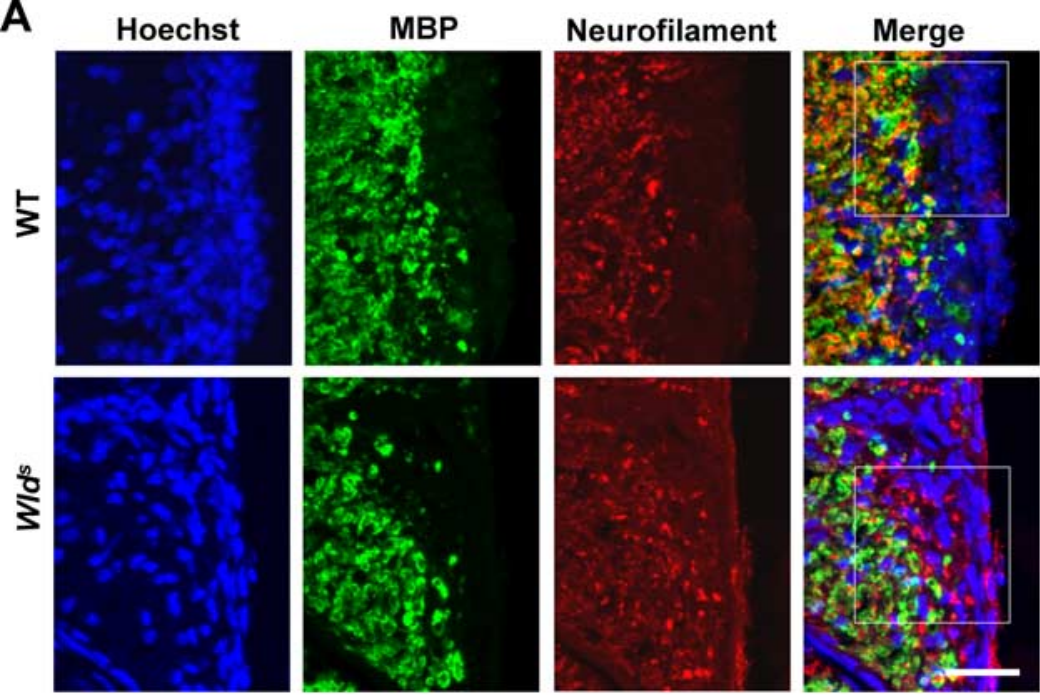

B

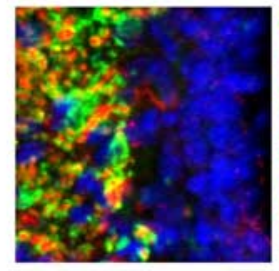

WT

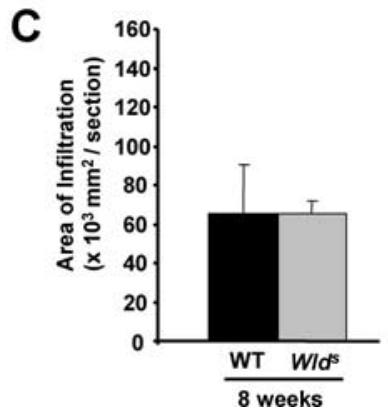

D

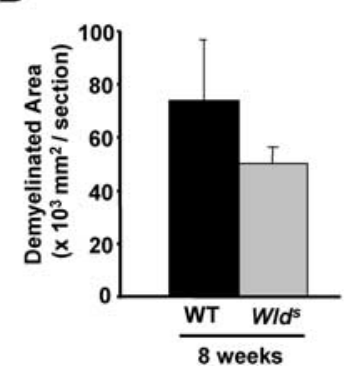

E

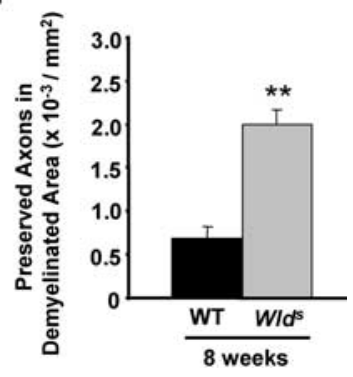

Figure 2. WId 5 expression reduces axon loss at 8 weeks p.i. $A$, Representative images of transverse sections from EAE-induced wild-type (top panels) and WId (bottom panels) mice at 8 weeks p.i. stained with Hoechst 33258 (left), anti-MBP (middle), or anti-NF (right). $\boldsymbol{B}$, The demyelinated areas are enlarged and the arrowheads indicate preserved axons in the demyelinated area. Scale bars: $\boldsymbol{A}, 30 \mu \mathrm{m} ; \boldsymbol{B}, 10 \mu \mathrm{m}$. $\boldsymbol{C}-\boldsymbol{E}$, Quantification of the average areas of infiltration $(p=0.99)(\boldsymbol{C})$ and demyelination $(p=$ $0.39)(D)$ per section, which showed no significant difference, and the average numbers of NF+/MBP - fibers in demyelinated areas, which was significantly increased in WId $d^{5}$ mice when compared with wild type ${ }^{* *} p<0.001$; Student's $t$ test) (E). Areas of infiltration $\left(\times 10^{3} \mu \mathrm{m}^{2} /\right.$ section $)$ or demyelination $\left(\times 10^{3} \mu^{2} /\right.$ section) were determined as the measured Hoescht + or $\mathrm{MBP}$ - areas averaged from 10 transverse sections per animal for each group. The number of preserved axons in demyelinated areas $\left(\times 10^{-3} / \mu \mathrm{m}^{2}\right)$ was determined by counting the number of NF+ puncta in demyelinated MBP - regions, and then dividing by the area of demyelination. Error bars indicate SEM.

\section{Modest protective effects of axon degeneration in $W l d^{s}$ mice}

We next assessed possible mechanisms involved in the protective effects of $W l d^{s}$ by immunohistochemistry analysis using animals that have been monitored for their neurological disabilities and terminated 8 weeks p.i. Our initial histological analysis indicated that the most severe plaques were seen most frequently in the brainstem and spinal cord (data not shown), consistent with previous studies (Onuki et al., 2001; Kalyvas and David, 2004; Karnezis et al., 2004). Thus, we focused our subsequent analyses on sections from cervical and lumbar spinal cord. Tissues from cer- vical spinal cord and one-half of the lumbar spinal cord were used for preparing serial transverse section, whereas the other one-half of the lumbar spinal cord was used for sagittal sections. A pilot study was performed to determine the appropriate number of sections for quantification to attain unbiased results considering the focal nature of the EAE model. Thus, for each animal, two sets of sections that cover the entire cervical and lumbar spinal cord (including 10 transverse sections: 5 from cervical and 5 from lumbar, each taken from every 10 sections; and 6 sagittal sections taken from every 5 sections) were stained with Hoechst 33258 to demonstrate cellular infiltration and simultaneously costained 
A Hoechst

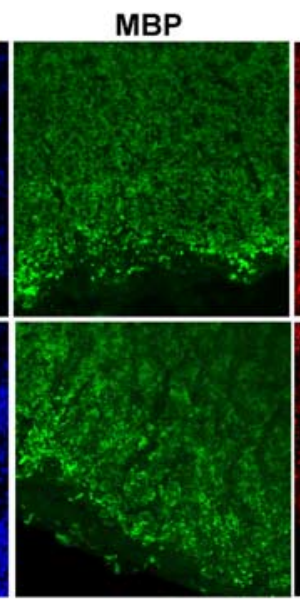

Neurofilament
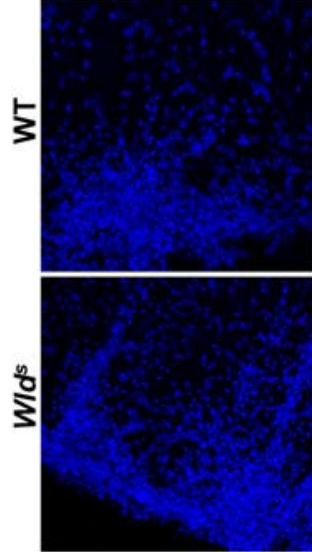

B

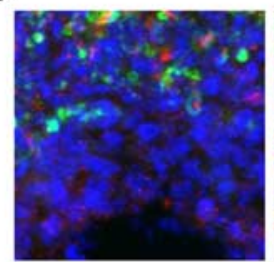

WT

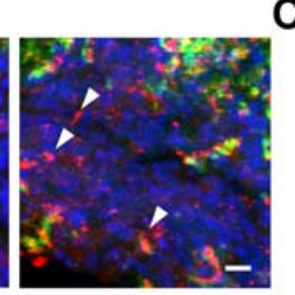

$W I d^{s}$

C
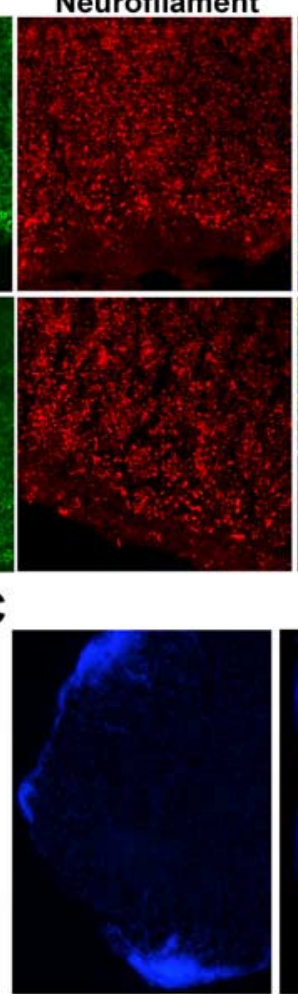

WT

$\mathbf{F}$

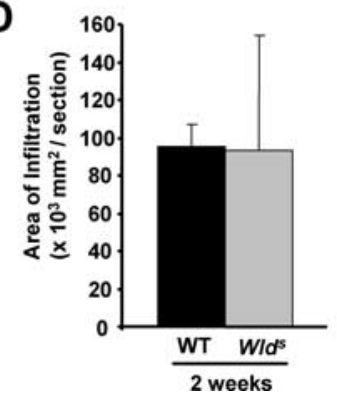

E

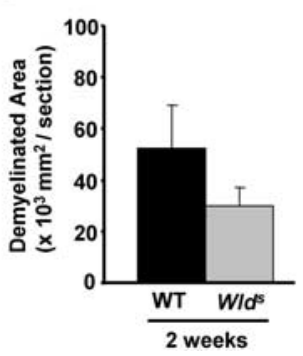

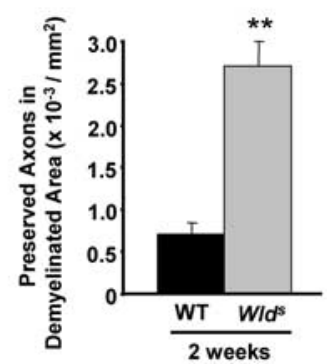

Figure 3. WId ${ }^{\mathrm{s}}$ expression reduces axon loss at 2 weeks p.i. $A-C$, Representative images of transverse sections from the lumbar spinal cords of EAE-induced wild-type or WId $d^{5}$ mice at 2 weeks p.i. Images of sections stained with Hoechst 33258/anti-MBP/NF $(\boldsymbol{A}$, $B$ ) show a similar extent of infiltration, but significantly more NF + fibers in the infiltrated and demyelinated areas from WId $5 A E$ mice compared with wild-type EAE mice. Images in $\boldsymbol{B}$ are enlarged from the indicated areas in $\boldsymbol{A}$. The arrowheads indicate several preserved axons in the lesion areas. Low magnification images of Hoechst 33258 staining indicating similar extents of infiltration in both wild-type and WId $d^{5}$ mice induced with EAE (C). Scale bars: $\boldsymbol{A}, \boldsymbol{C}, 100 \mu \mathrm{m} ; \boldsymbol{B}, 10 \mu \mathrm{m}$. D-F, Quantification of the average areas of infiltration $(p=0.97)(\boldsymbol{D})$ and demyelination $(p=0.25)(\boldsymbol{E})$ per section, which showed no significant difference, and the average numbers of NF $+/ M B P$ - fibers in demyelinated areas, which was significantly increased in WId ${ }^{5}$ mice compared with wild type ${ }^{* *} p<0.001$; Student's $t$ test) $(\boldsymbol{F})$. Error bars indicate SEM.

with antibodies to MBP and NF200 to show the areas of demyelination and axonal loss, respectively.

Immunostaining results were generally similar in both transverse and sagittal sections, although quantifications were performed from transverse sections, where both the size of lesioned areas and axonal density can be more appropriately measured. As shown in Figure 2, $A$ and $C$, the average size of infiltrated areas per section as shown by Hoeschst 33258 staining was similar between wild-type and Wld mice. The area of demyelination, as determined from anti-MBP staining, was smaller in $W l d^{s}$ mice than their wild-type controls, although the difference was not statistically significant ( $p=0.39$ ) (Fig. $2 A, D$ ). In contrast, we observed a significant difference in the extent of axonal loss between these

Merge
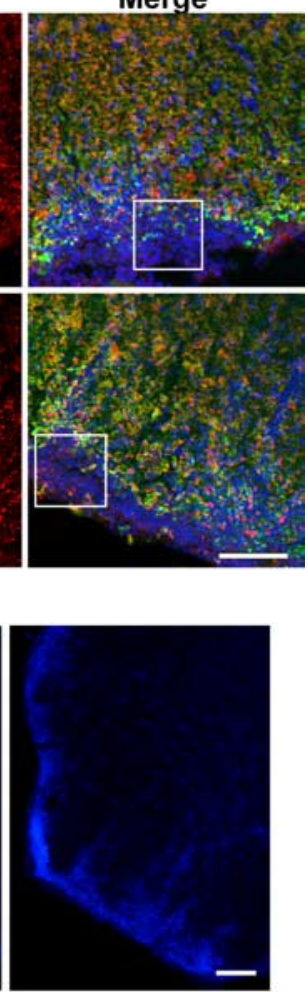

WIds two groups. Within the demyelinated areas in control EAE animals, few axons were positively labeled with anti-NF antibodies (Fig. $2 A, B, E$ ). On the other hand, we found significantly increased numbers of $\mathrm{NF}+$ and $\mathrm{MBP}-$ axons within the demyelinated areas in sections from $W l d^{s}$ animals induced with EAE (Fig. $2 A, B, E$ ). Although we cannot rule out the possibility of protection from inflammatory demyelination, our results suggest that overexpressed $\mathrm{Wld}^{\mathrm{s}}$ are primarily responsible for preserving demyelinated axons in the EAE model.

As our behavioral results suggest that Wld $^{s}$ also alleviates the neurological disability even during the early phase of the EAE model (Fig. $1 \mathrm{~A}$ ), we decided to analyze the histological basis of $\mathrm{Wld}^{\mathrm{s}}$ mediated protection under these conditions (Fig. 1A). Thus, we induced a separate set of wild-type and $W l d^{s}$ mice with EAE (eight animals per group), which all developed behavioral deficits similar to those shown in Figure $1 \mathrm{~A}$ (data not shown). These animals were terminated at 2 weeks p.i. for histological analysis as described above. The average infiltrated areas were larger than those from 8 weeks p.i. (compare Figs. 3D, 2C), suggesting that the extent of infiltration decreases in the chronic phase of EAE. Similar to the results from EAE animals examined at 8 weeks p.i., the difference in the areas of infiltration and demyelination were not statistically significant between wild-type and $W l d^{s}$ groups (Fig. $3 A, D, E$ ). Interestingly, we found that even at 2 weeks p.i., few NF+ fibers could be seen within the infiltrated and demyelinated areas (Fig. $3 A, B, F)$, indicating the presence of significant axonal damage even at the early phase of the EAE model. Consistent with this, we found that the corresponding staining on sagittal sections showed residual fibers, some with varicosities (Fig. 4A) or endings (Fig. $4 B$ ), within the demyelinated areas. In addition, electron microscopic examination confirmed the existence of degenerating axons within the lesions of EAE animals (Fig. 4D). On the other hand, the number of demyelinated but preserved (MBP-/NF+) fibers was significantly higher in sections from EAE-induced $W l d^{s}$ mice (Fig. $3 F$ ). Together, our results suggest that the axonal loss that occurs during both the acute and chronic phases of EAE are significantly reduced in $W l d^{s}$ mice.

\section{Insignificant effects of Wld ${ }^{s}$ on immune infiltration in the EAE model}

The results of Hoechst staining suggested that the extent of infiltration in either 2 or 8 weeks p.i. of EAE models were not significantly altered by Wld $^{\text {s }}$ (Figs. 2C, 3D). To further examine whether specific types of infiltrated immune cells may be affected, 
we performed immunostaining on sagittal sections from EAE-induced animals at 2 weeks p.i. with anti-CD4 antibodies for identifying infiltrated helper $\mathrm{T}$ cells (Tompkins et al., 2002). The numbers of infiltrated CD4+ cells were quantified by coimmunostaining nuclei by Hoechst 33258 by performing high-powered microscopic analysis. As shown in Figure $5 A-C$, the numbers of CD4+ were comparable in sections from wild-type and $W l d^{s}$ mice. Similar results have been obtained with immunostaining with anti-CD8 antibodies for cytotoxic $\mathrm{T}$ cells (data not shown). These results suggested that the infiltration of $\mathrm{T}$ cells is not significantly affected by Wld ${ }^{\mathrm{s}}$ expression.

We also examined whether the extent of cell death that occurs during the acute phase of EAE is affected by Wld ${ }^{\text {s. Once }}$ again, we failed to observe any significant difference between wild-type and $W l d^{s}$ mice in the number of total apoptotic cells, as detected by TUNEL (Fig. 5D,E). These results together suggest that $\mathrm{Wld}^{\mathrm{s}}$ mediated alleviation of neurological disability may primarily result from its protective effects on axonal damage.

\section{Prevention of NAD decline in $W l d^{s}$ - based EAE model}

As our previous studies suggested that neuronal NAD levels decreases in axons undergoing Wallerian degeneration in vitro (Wang et al., 2005), we next examined whether there is a decrease in NAD levels in the CNS of EAE animals. Extracts were prepared from segments of cervical spinal cord dissected from EAE-induced wild-type and $W l d^{s}$ mice for measuring NAD levels using reverse-phase HPLC (Wang et al., 2005). As shown in Figure $1 C$, in contrast to the progressive and statistically significant decline in NAD levels in tissues from control EAE models at 2 and 4 weeks p.i., NAD levels were preserved at corresponding time points in $W l d^{s}$ animals (Fig. 1C). Considering the focal nature of EAE lesions, the measured NAD levels may even underestimate the extent of NAD decline in degenerating axons. Nevertheless, our results suggest that Wld ${ }^{\mathrm{s}}$ may protect axons from degeneration by preventing NAD decline in the CNS of the EAE model.

Profound protective effects of NAm on neurological disability in the EAE model

It is possible that the modest protective effect of $\mathrm{Wld}^{\mathrm{s}}$ may relate to the observation that NAD levels are not increased in tissues of Wld $d^{s}$ mice (Mack et al., 2001). In this regard, our previous studies suggested that, when added to the medium, NAm, the amide form of niacin (vitamin B3), increases NAD levels in cultured neurons (Wang et al., 2005). Thus, to bolster NAD levels, we administered NAm at two different doses (125 or $500 \mathrm{mg} / \mathrm{kg}$ ) by daily subcutaneous injection starting from the day of MOG inoculation. As control, we administered the same volume of PBS into either wild-type or $W l d^{s}$ mice.

As shown in Figure 6C, daily injection of NAm dramatically increased NAD levels in a dose-dependent manner, as measured at 2 weeks p.i. However, despite continuous daily administration,
NAD levels returned to near-normal levels in wild-type mice at 4 weeks p.i. In contrast, in $W l d^{s}$ mice with high dose NAm administration, NAD levels remained high at both 2 and 4 weeks p.i. (Fig. 6C), presumably because of the ability of Wld ${ }^{\text {s}}$-associated Nmnat 1 to facilitate the conversion of NAm to NAD. It is currently unclear why the increased NAD levels returned to baseline after 4 weeks of NAm administration. One possibility is that homeostatic mechanisms may act to maintain a normal level of NAD in these cells.

Strikingly, we observed that the protective effects of NAm and/or Wld ${ }^{s}$ on neurological disability closely correlate with changes in NAD levels as described above. In wild-type mice, NAm ameliorated the onset and development of EAE-associated deficits in a dose-dependent manner (Fig. 6A,B). Whereas lowdose NAm administration had a similar effect as Wld ${ }^{\text {s }}$ expression (compare Figs. 6A, 1A), high-dose NAm treatment showed a significantly greater protective effect by both delaying the onset of the disease (Fig. 6B) and reducing the severity of deficits for at least 8 weeks (Fig. 6A). The most dramatic protection was observed in $W l d^{s}$ mice with high-dose NAm treatment (Fig. 6A,B). Interestingly, at 8 weeks p.i., differences in behavioral scores could be seen between NAm-treated wild-type and $W l d^{s}$ mice (Fig. 6A), which correlate with the observed NAD levels in these groups (Fig. 6C).

Protective effects of NAm on inflammation, demyelination, and axonal loss

We next examined the histological basis of NAm-mediated protection by Hoechst 33258/MBP/NF costaining (at both 2 and 8 weeks p.i.), as well as anti-CD4 (at 2 weeks p.i.), following the procedures described above. Unlike the effects of Wld ${ }^{\mathrm{s}}$, NAm treatment reduced the areas of infiltration and demyelination 
A

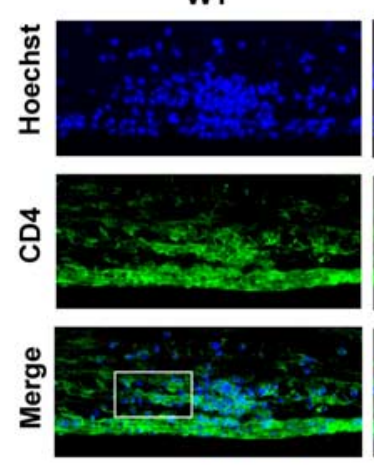

D
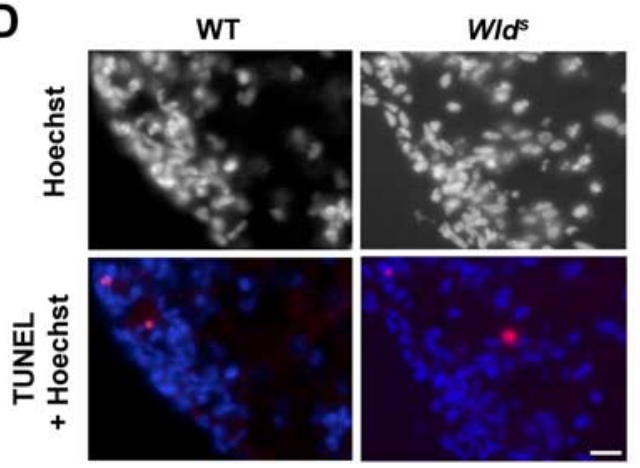

\section{WIds}

B
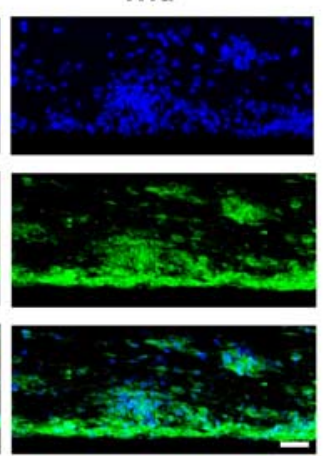

$\mathbf{E}$

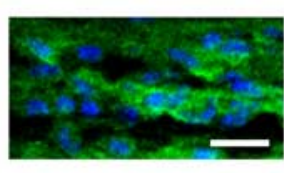

C
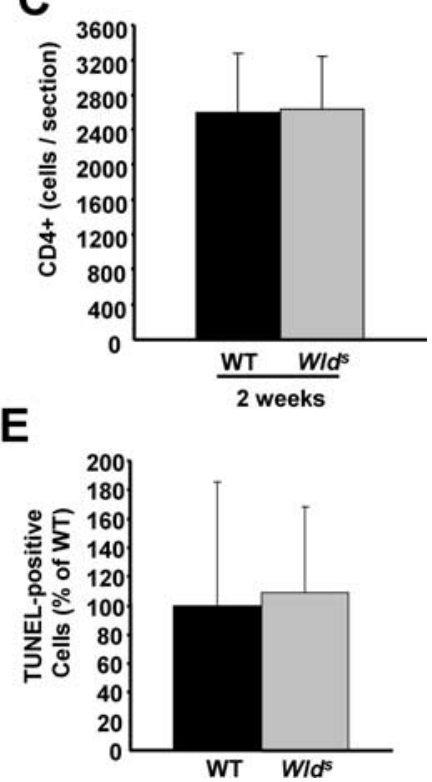

Figure 5. The effects of WId ${ }^{s}$ on infiltration of immune cells and cell death in the EAE model. Sagittal sections from 2 weeks p.i. EAE-induced wild-type or WId mice were stained with Hoechst 33258 and antibodies against CD4 (A). Representative images are shown in $\boldsymbol{A}$. The panel shown in $\boldsymbol{B}$ is an enlargement of the selected area of merged image. Scale bars: $\boldsymbol{A}, 25 \mu \mathrm{m}$; enlarged panel, $10 \mu \mathrm{m}$. Quantification of the average number of infiltrated CD4 $+(C)$ cells (numbers per section) from six sections per animals, eight animals per group, showing no significant differences in these groups ( $p=0.97$; Student's $t$ test). $\boldsymbol{D}$, Representative images of TUNEL (red in bottom panel) costained with Hoechst 33258 (white in top panel and blue in bottom panel) in the lesion areas of the cervical spinal cord transverse sections of wild-type (left panels) or WId (right panels) mice at 2 weeks p.i. Scale bars, $15 \mu \mathrm{m}$. $E$, Quantification of TUNEL-positive cells in EAE-induced wild-type and WId ${ }^{5}$ mice ( 10 sections per animal from 8 animals in each group) at 2 weeks p.i., showing no significant difference in these groups ( $p=0.93$; Student's $t$ test). Error bars indicate SEM.

(Fig. $7 A-D$ ) as well as the number of infiltrated CD4+ (Fig. $7 F$ ) in both wild-type and Wld mice, suggesting that NAm has a protective effect against inflammation and demyelination. Previous studies have shown that NAm can prevent endothelial cell injury under conditions such as ischemia (Chong et al., 2002). Thus, it is possible that the observed effects of NAm on inflammation and demyelination may result from its effects on endothelial cells, thus resulting in reduced inflammatory infiltration and subsequent demyelination. However, it is unknown whether this effect may also be NAD dependent.

Importantly, we also observed a significant increase in the numbers of NF+/MBP - axons within the demyelinated lesions in NAm-treated EAE animals, which was even further increased in the NAm-treated $W l d^{s}$ mice induced with EAE (Fig. $7 A, B, E$ ). In sections from Wld $d^{s}$ mice induced with EAE and treated with NAm, the density of preserved axons in the demyelinated regions was estimated to reach at least $70 \%$ of the density in the surrounding nonlesion region (Fig. $7 A, B$ ), suggesting a strong neuroprotective effect with NAm administration. Thus, although the protective effects of NAm on inflammatory demyelination certainly contribute to the improvement in behavior, our results suggest that NAm likely acts to protect against axon degeneration in addition to its antiinflammation/demyelination activity.
NAm attenuates axon damage by LPSactivated microglia in vitro

To directly examine whether NAD increase by NAm could protect against the axonal damage triggered by inflammation, we used an in vitro model for microgliamediated neurotoxicity (Lehnardt et al., 2003). As shown in Figure 8, the number of Tuj1-positive cortical neurons decreased significantly after exposure to LPS-activated microglia. However, NAm treatment could profoundly attenuate the neurotoxicity triggered by LPS-activated microglia. This protective effect of NAm was obvious even when NAm was only used to pretreat cultured neurons before coculture with activated microglia. Thus, together with the in vivo data shown in Figure 7 , these results suggest that NAm has a direct neuroprotective role.

Delayed NAm treatment attenuates EAE To further explore the possibility of using NAm as a therapeutic means to treat animals that have already developed EAE, we examined whether delayed NAm administration would also exhibit protective effects. We started daily treatments with NAm $(500 \mathrm{mg} / \mathrm{kg})$ from $10 \mathrm{~d}$ p.i. when most of the animals have already displayed clear signs of neurological disability (Fig. 9A). In these animals, NAm treatment efficiently attenuated additional development of EAE symptoms (Fig. 9A). As expected, this treatment also effectively increased NAD levels (Fig. 9B). At 8 weeks p.i., whereas the areas of inflammation and demyelination were modestly reduced (Fig. 10A-D), axonal loss was significantly prevented (Fig. $10 A, B, E$ ). In addition, the protective effects of NAm are not limited to the MOG peptideinduced EAE model, because NAm administration also significantly attenuates the behavioral deficits in another frequently used EAE model that involves induction by the $\mathrm{PLP}_{139-151}$ peptide in $(\mathrm{PL} / \mathrm{J} \times \mathrm{SJL} / \mathrm{J}) \mathrm{F}_{1}$ mice (data not shown). Together, these results suggest the possibility of using NAm as a therapeutic measure for even the chronic stages of EAE.

\section{Discussion}

In this study, we showed that both Wld ${ }^{\mathrm{s}}$ expression and NAm administration can prevent axonal loss and alleviate the neurological disability associated with EAE, suggesting a new neuroprotective strategy for MS/EAE. Although NAm also shows a protective effect on inflammation and demyelination, we found that $\mathrm{Wld}^{s}$ and/or NAm resulted in increased numbers of demyelinated but preserved axons within EAE lesions, substantiating the notion that these treatments could protect demyelinated axons from additional degeneration. The extent of axon protection correlates with the neurological scores from these groups, further supporting a critical contribution of axonal damage to EAEassociated behavioral deficits. In addition, our results indicate that significant axonal damage and loss occur in the EAE model at 


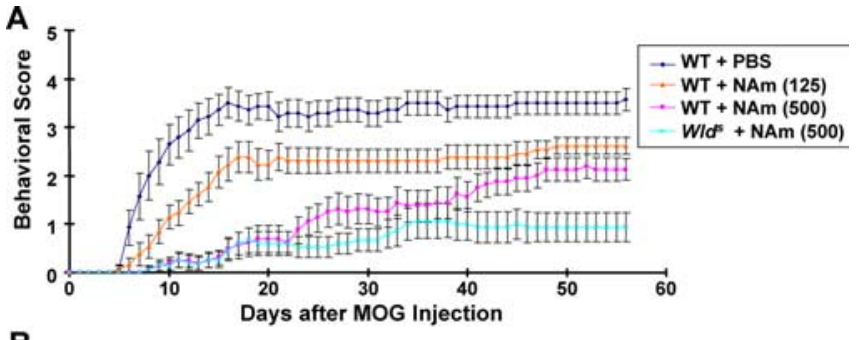

B

\begin{tabular}{|l|c|c|c|c|}
\hline & $W T+$ PBS & $\begin{array}{c}\text { WT + NAm } \\
(125 \mathrm{mg} / \mathrm{kg})\end{array}$ & $\begin{array}{c}\text { WT + NAm } \\
(500 \mathrm{mg} / \mathrm{kg})\end{array}$ & $\begin{array}{c}\text { W/d }+\mathrm{NAm} \\
(500 \mathrm{mg} / \mathrm{kg})\end{array}$ \\
\hline Incidence & $14 / 14$ & $13 / 13$ & $15 / 16$ & $10 / 15$ \\
\hline Death from Severe Disease & 3 & 0 & 0 & 0 \\
\hline Onset Day & $7.5 \pm 0.6$ & $9.7 \pm 0.9^{*}$ & $26.4 \pm 3.8^{* *}$ & $34.5 \pm 5.8^{* *}$ \\
\hline
\end{tabular}

C

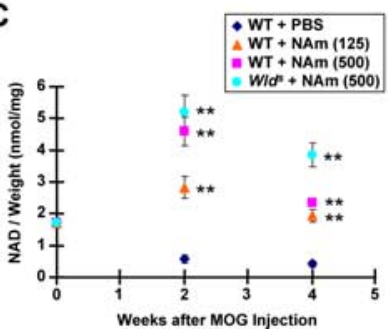

Figure 6. Profound protective effects of NAm on the behavioral defects of the EAE model. $\boldsymbol{A}$, Behavioral scores (mean $\pm \mathrm{SEM}$ ) of different groups of EAE mice. Differences between each treated group and untreated controls were significant as determined by two-tailed Student's $t$ test: $p<0.05$, from $7 \mathrm{~d}$ p.i. in the low-dose NAm-treated wild-type group $(125 \mathrm{mg} / \mathrm{kg}) ; p<$ 0.001 , from $6 \mathrm{~d}$ p.i. in the high-dose NAm-treated wild-type and W/d groups $(500 \mathrm{mg} / \mathrm{kg}) . \boldsymbol{B}$, Clinical features of the EAE model in NAm-treated groups and untreated controls. Symptom onset was significantly delayed in each treated group when compared with untreated wild-type EAE mice $\left({ }^{*} p<0.05 ;{ }^{* *} p<0.001\right.$; Student's $t$ test). C, NAD levels (mean \pm SEM) in cervical spinal cords from different groups of EAE mice as analyzed by HPLC. At both 2 and 4 weeks p.i., the NAD levels in each treated group were significantly higher than those from untreated controls $\left({ }^{* *} p<0.001\right.$; Student's $t$ test).

2 weeks p.i., suggesting the necessity of applying neuroprotective strategies during the early stage of MS/EAE.

\section{Mechanisms of axon degeneration in MS/EAE}

Both Wld ${ }^{\mathrm{s}}$ expression and NAm administration reduce axon degeneration in EAE models, suggesting that the axon damage that occurs in the CNS of the EAE model may be mechanistically similar to Wallerian degeneration. However, the direct triggers leading to axonal destruction in MS lesions are still under debate. It has been proposed that axon damage could result from direct immunologic attack on axons by cytotoxic $\mathrm{T}$ lymphocytes or soluble inflammatory mediators (Neumann et al., 2002), or be secondary to inflammatory demyelination (Steinman, 2001). Our demonstration that Wld ${ }^{\mathrm{s}}$ and NAm increase the numbers of demyelinated but preserved axons provides direct support for the latter possibility. Thus, it is possible that, in EAE/MS lesions, demyelinated axons may become vulnerable to damage by such inflammatory mediators as proteolytic enzymes, cytokines, oxidative products, and free radicals that are produced by activated immune and glial cells, as proposed previously (Stys, 2005; Waxman et al., 2005).

\section{NAD decrease in EAE models}

Similar to the decrease in NAD levels in degenerating axons of cultured neurons after mechanical or chemical insults (Wang et al., 2005), we found a similar decline in NAD levels in the spinal cord of EAE animals. Considering the focal nature of EAE lesions, the observed decrease in NAD levels may underestimate the ex- tent of decline that actually occurs in degenerating axons. However, the precise mechanisms leading to this NAD decrease in EAE lesions remain unclear. One possibility is that inflammatory mediators such as oxidative products and free radicals could activate neuronal NAD-consuming molecules including poly(ADP-ribose) polymerases (PARP), Sirt family members, and/or cADPR (cyclic adenosine diphosphoribose) (Berger et al., 2004). PARP1, for example, has been shown to be activated in both neurons and glial cells in a nonhuman primate EAE model (Kauppinen et al., 2005). Although PARP1 is a nuclear protein, other PARP proteins have also been shown to be localized to the cytoplasmic compartment (Ha and Snyder, 2000). Thus, the involvement of these and other enzymes in consuming NAD in MS/EAE lesions remains to be determined.

How does NAD protect axons from degeneration? Our previous results suggest that the decrease in NAD may impair local energy metabolism in the axon, ultimately leading to axon degeneration (Wang et al., 2005). In the same line, we found that increasing NAD levels could attenuate the neuronal toxicity induced by LPS-activated microglia. This is consistent with a recent demonstration that mitochondrial dysfunction and energy failure may be a cause of axonal degeneration in MS patients (Dutta et al., 2006). Thus, it is possible that both Wld ${ }^{s}$ and NAm could improve the local bioenergetics, thus acting to protect axons from degeneration in the EAE plaques. Interestingly, infiltrated microglial cells were decreased in both Wld ${ }^{s}$-based EAE models and NAm-treated models (T. Chitnis and S. J. Khoury, unpublished observations; our unpublished observations). In light of ample evidence suggesting that axons undergoing Wallerian degeneration could trigger macrophage/microglia responses (George and Griffin, 1994), it is also possible that protected axonal degeneration leads to less microglial infiltration and activation, which may also contribute to the extent of axonal loss in the CNS of EAE models.

\section{Nicotinamide as a therapeutic agent}

In the past, efforts have been made to examine the possible neuroprotective effects of a variety of agents using EAE models, including cytokines, growth factors, and blockers of voltage-gated sodium channels (for review, see Stys, 2005; Waxman, 2005). Our results suggest a novel potential therapeutic avenue using protective strategies based on studies of Wallerian degeneration to prevent axon damage in MS and EAE models. The observed correlations between the effects of increasing NAD levels, preventing axon damage, and alleviating behavioral deficits, offer a potential rationale for designing efficient protective strategies for EAE/MS. In particular, NAm can prevent the decline of NAD levels in the CNS tissues of EAE animals, and also confer a profound protective effect on axon degeneration in the EAE model. Interestingly, NAm also reduces the extent of inflammation and demyelination in EAE animals, which may be explained by the ability of NAm to protect endothelial cells from injury (Chong et al., 2002; Klaidman et al., 2003; Maiese and Chong, 2003), thus reducing inflammatory infiltration and subsequent demyelination. Although we cannot rule out other possibilities, it is possible that the protective effects of NAm on neurons, endothelial cells, and perhaps oligodendrocytes reflect the influence of different NAD levels resulting from Wld ${ }^{s}$ overexpression and/or NAm treatment, and neurons and non-neuronal cells may have different sensitivities to the cellular NAD levels.

In addition to its clear protective effects, NAm can readily cross the blood-brain barrier and plasma membranes (Hankes et al., 1991; Maiese and Chong, 2003), and may thus be a promising 

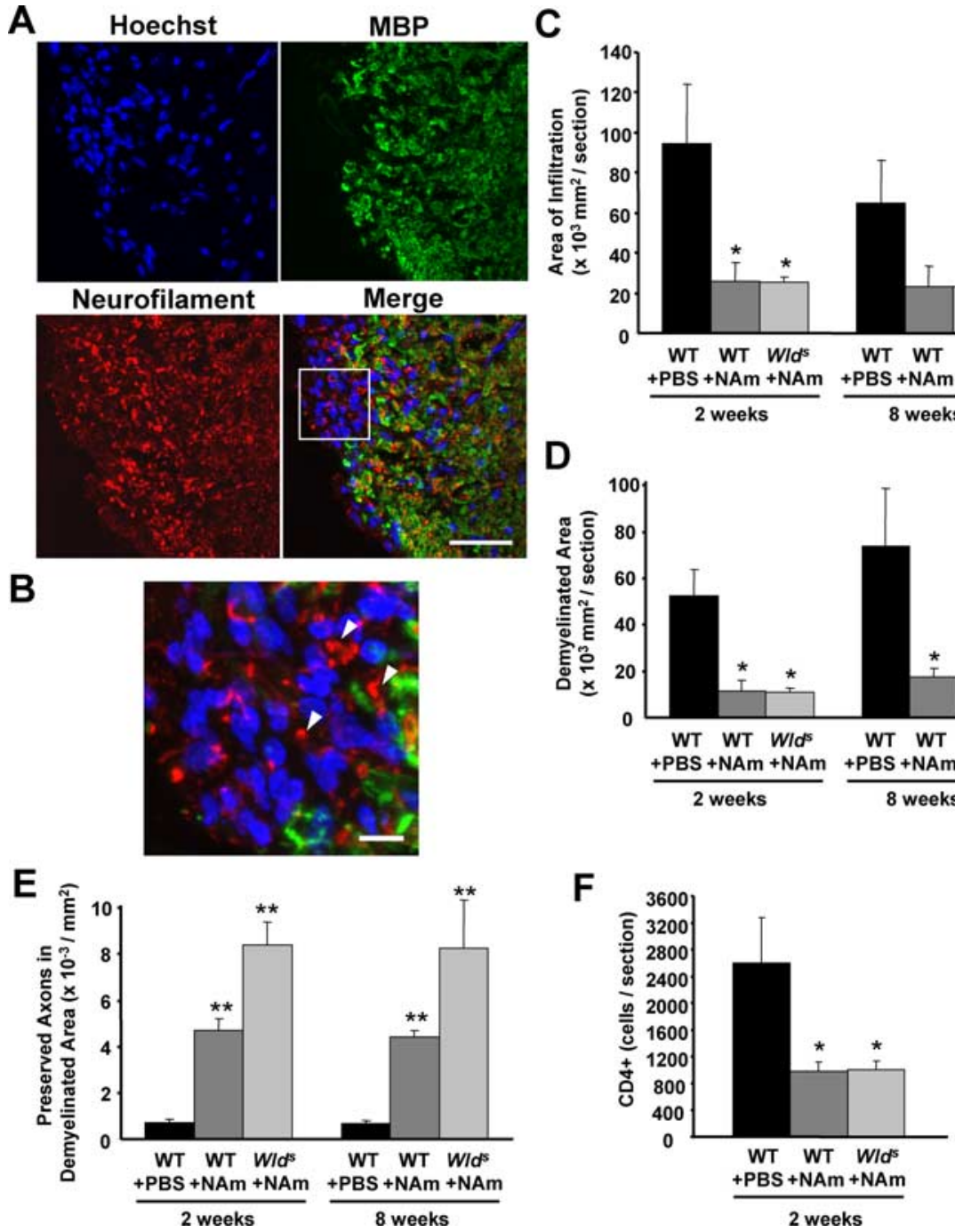

Figure 7. Profound protective effects of NAm on inflammation, demyelination and axonal loss in the EAE model. $\boldsymbol{A}, \boldsymbol{B}$, Representative images showing the effects of NAm treatment on infiltration, demyelination, and axonal loss. Transverse sections from 8 weeks p.i. EAE-induced WId $d^{5}$ mice treated with high-dose NAm were stained with Hoechst 33258 and antibodies against MBP and NF $(\boldsymbol{A})$. Higher magnification of the merged image is shown in $\boldsymbol{B}$. Scale bars: $\boldsymbol{A}, 50 \mu \mathrm{m} ; \boldsymbol{B}, 10 \mu \mathrm{m}$. $\boldsymbol{C}-\boldsymbol{E}$, Quantification of average areas of infiltration $(\boldsymbol{C}$ ) and demyelination $(\boldsymbol{D})$, as well as average number of NF $+/ \mathrm{MBP}$ - fibers in demyelinated areas (E). Both NAm-treated groups showed a significant reduction in infiltration at 2 weeks p.i. $\left({ }^{*} p<0.05\right)$, and demyelination at both 2 and 8 weeks p.i. ( $\left.{ }^{*} p<0.05\right)$, but not infiltration at 8 weeks p.i. ( $p=0.11, p=0.10$ in wild-type and WId ${ }^{5}$ mice, respectively) $(\boldsymbol{C}, \boldsymbol{D})$. Average numbers of preserved fibers in demyelinated areas were also significantly increased in both NAm-treated groups at both time points $\left({ }^{* *} p<0.001\right.$; Student's $t$ test $)(\boldsymbol{E})$. Quantification of the infiltrated CD4+ cells $(\boldsymbol{F})$, showing a significant reduction of $C D 4+$ cellular infiltration in both NAm-treated groups $\left({ }^{*} p<0.05\right.$; Student's $t$ test). Average immune cell numbers per section from six sections of each animal, eight animals per group, were quantified. Error bars indicate SEM.

candidate as a neuroprotective treatment for MS patients. Interestingly, we found that, although NAm administration could increase NAD levels during the first 2 weeks after EAE induction, the increased NAD levels returned to relatively normal levels after 4 weeks. However, NAm administration in $W l d^{s}$ mice could maintain a higher level of NAD for at least 4 weeks, presumably because of a more efficient conversion of NAm to NAD. Thus, better understanding the homeostatic regulation of neuronal NAD levels may allow us to design the means to further enhance the protective effects of NAm for MS/EAE and perhaps other axon degeneration-relevant diseases.

\section{References}

Adelman B, Sandrock A, Panzara MA (2005) Natalizumab and progressive multifocal leukoencephalopathy. N Engl J Med 353:432-433.
Aktas O, Smorodchenko A, Brocke S, InfanteDuarte C, Topphoff US, Vogt J, Prozorovski T, Meier S, Osmanova V, Pohl E, Bechmann I, Nitsch R, Zipp F (2005) Neuronal damage in autoimmune neuroinflammation mediated by the death ligand TRAIL. Neuron 46:421-432.

Araki T, Sasaki Y, Milbrandt J (2004) Increased nuclear NAD biosynthesis and SIRT1 activation prevent axonal degeneration. Science 305:1010-1013.

Berger F, Ramirez-Hernandez MH, Ziegler M (2004) The new life of a centenarian: the new life of a centenarian: signalling functions of NAD(P). Trends Biochem Sci 29:111-118.

Bjartmar C, Wujek JR, Trapp BD (2003) Axonal loss in the pathology of MS: consequences for understanding the progressive phase of the disease. J Neurol Sci 206:165-171.

Chong ZZ, Lin SH, Maiese K (2002) Nicotinamide modulates mitochondrial membrane potential and cysteine protease activity during cerebral vascular endothelial cell injury. J Vasc Res 39:131-147.

Coleman M (2005) Axon degeneration mechanisms: commonality amid diversity. Nat Rev Neurosci 6:889-898.

Dutta R, McDonough J, Yin X, Peterson J, Chang A, Torres T, Gudz T, Macklin WB, Lewis DA, Fox RJ, Rudick R, Mirnics K, Trapp BD (2006) Mitochondrial dysfunction as a cause of axonal degeneration in multiple sclerosis patients. Ann Neurol 59:478-489.

Ferguson B, Matyszak MK, Esiri MM, Perry VH (1997) Axonal damage in acute multiple sclerosis lesions. Brain 120:393-399.

George R, Griffin JW (1994) Delayed macrophage responses and myelin clearance during Wallerian degeneration in the central nervous system: the dorsal radiculotomy model. Exp Neurol 129:225-236.

Ha HC, Snyder SH (2000) Poly(ADP-ribose) polymerase- 1 in the nervous system. Neurobiol Dis 7:225-239.

Hankes LV, Coenen HH, Rota E, Langen KJ, Herzog H, Wutz W, Stoecklin G, Feinendegen LE (1991) Effect of Huntington's and Alzheimer's diseases on the transport of nicotinic acid or nicotinamide across the human bloodbrain barrier. Adv Exp Med Biol 294:675-678.

Kalyvas A, David S (2004) Cytosolic phospholipase A2 plays a key role in the pathogenesis of multiple sclerosis-like disease. Neuron 41:323-335.

Karnezis T, Mandemakers W, McQualter JL, Zheng B, Ho PP, Jordan KA, Murray BM, Barres B, Tessier-Lavigne M, Bernard CC (2004) The neurite outgrowth inhibitor Nogo A is involved in autoimmune-mediated demyelination. Nat Neurosci 7:736-744.

Kauppinen TM, Suh SW, Genain CP, Swanson RA (2005) Poly(ADPribose) polymerase-1 activation in a primate model of multiple sclerosis. J Neurosci Res 8:190-198.

Klaidman L, Morales M, Kem S, Yang J, Chang ML, Adams Jr JD (2003) Nicotinamide offers multiple protective mechanisms in stroke as a precursor for $\mathrm{NAD}+$, as a PARP inhibitor and by partial restoration of mitochondrial function. Pharmacology 69:150-157.

Lehnardt S, Massillon L, Follett P, Jensen FE, Ratan R, Rosenberg PA, Volpe JJ, Vartanian T (2003) Activation of innate immunity in the CNS triggers neurodegeneration through a Toll-like receptor 4-dependent pathway. Proc Natl Acad Sci USA 100:8514-8519.

Luo L, O'Leary DD (2005) Axon retraction and degeneration in development and disease. Annu Rev Neurosci 28:127-156. 
Mack TG, Reiner M, Beirowski B, Mi W, Emanuelli M, Wagner D, Thomson D, Gillingwater T, Court F, Conforti L, Fernando FS, Tarlton A, Andressen C, Addicks K, Magni G, Ribchester RR, Perry VH, Coleman MP (2001) Wallerian degeneration of injured axons and synapses is delayed by a Ube $4 \mathrm{~b} / \mathrm{Nmnat}$ chimeric gene. Nat Neurosci 4:1199-1206.

Maiese K, Chong ZZ (2003) Nicotinamide: necessary nutrient emerges as a novel cytoprotectant for the brain. Trends Pharmacol Sci 24:228-232.

Matthews PM, De Stefano N, Narayanan S, Francis GS, Wolinsky JS, Antel JP, Arnold DL (1998) Putting magnetic resonance spectroscopy studies in context: axonal damage and disability in multiple sclerosis. Semin Neurol 18:327-336.

Neumann H, Medana IM, Bauer J, Lassmann H (2002) Cytotoxic T lymphocytes in autoimmune and degenerative CNS diseases. Trends Neurosci 25:313-319.

Onuki M, Ayers MM, Bernard CC, Orian JM (2001) Axonal degeneration is an early pathological feature in autoimmune-mediated demyelination in mice. Microsc Res Tech 52:731-739.

Raff MC, Whitmore AV, Finn JT (2002) Axonal self-destruction and neurodegeneration. Science 296:868-871

Steinman L (2001) Multiple sclerosis: a twostage disease. Nat Immunol 2:762-764.

Stys PK (2005) General mechanisms of axonal damage and its prevention. J Neurol Sci 233:3-13.

Tompkins SM, Padilla J, Dal Canto MC, Ting JP, Van Kaer L, Miller SD (2002) De novo central nervous system processing of myelin antigen is required for the initiation of experimental autoimmune encephalomyelitis. J Immunol 168: 4173-4183.

Trapp BD, Peterson J, Ransohoff RM, Rudick R, Mork S, Bo L (1998) Axonal transection in the lesions of multiple sclerosis. N Engl J Med 338:278-285.

Wang J, Zhai Q, Chen Y, Lin E, Gu W, McBurney MW, He Z (2005) A local mechanism mediates NAD-dependent protection of axon degeneration. J Cell Biol 170:349-355.

Waxman SG (2005) Sodium channel blockers and axonal protection in neuroinflammatory disease. Brain 128:5-6.

Zhai Q, Wang J, Kim A, Liu Q, Watts R, Hoopfer E, Mitchison T, Luo L, He Z (2003) Involvement of the ubiquitin-proteasome system in the early stages of wallerian degeneration. Neuron 39:217-225.

Zhu B, Guleria I, Khosroshahi A, Chitnis T, Imitola J, Azuma M, Yagita H, Sayegh MH, Khoury SJ (2006) Differential role of programmed death-1

B indicate SEM.
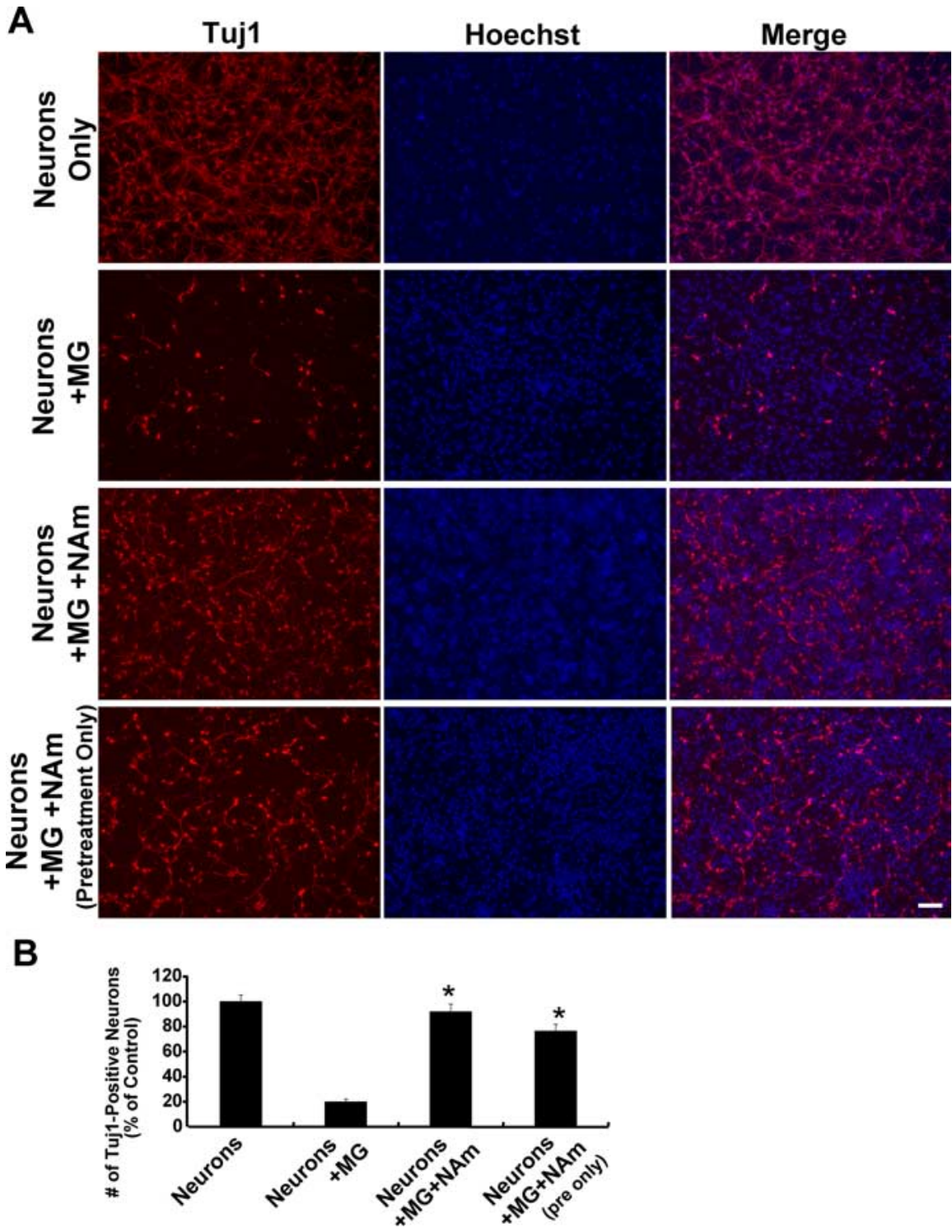

Figure 8. NAm attenuates neurotoxicity induced by LPS-activated microglia. $\boldsymbol{A}$, Representative images showing cocultures of E18 cortical neurons and LPS-activated microglia in different conditions stained with Tuj-1 antibody or Hoechst 33258. Images of cultured neurons without microglia (first row), with microglia (second row), with microglia plus $25 \mathrm{~mm}$ NAm (third row), and with microglia plus $25 \mathrm{~mm}$ NAm (pretreatment only) (fourth row). Scale bar, $100 \mu \mathrm{m}$. B, Quantification of Tuj-1-positively stained neurons under different conditions. NAm treatments (both continuous or pretreatment alone) significantly preserved neurons cocultured with LPS-activated microglia ( ${ }^{*} p<0.001$; Student's $t$ test). Error bars

ligand and programmed death-2 ligand in regulating the susceptibility and chronic progression of experimental autoimmune encephalomyelitis. J Immunol 176:3480-3489. 
A

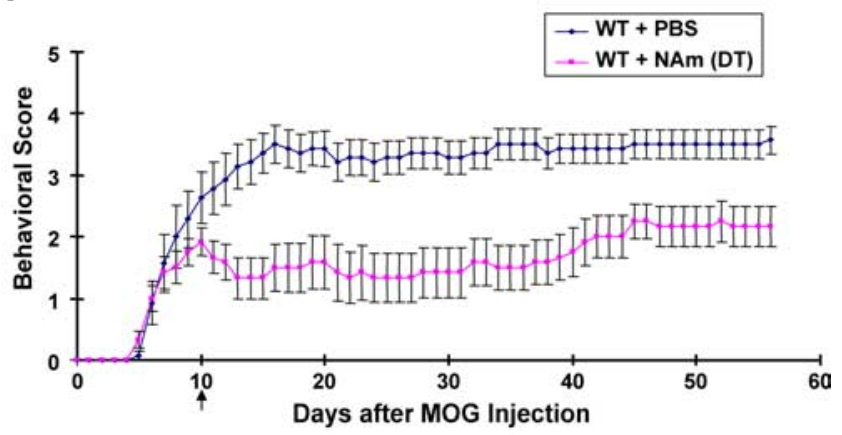

B

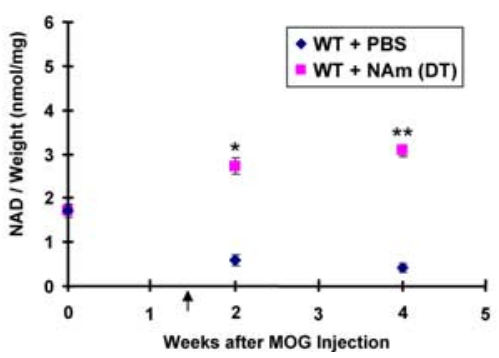

Figure 9. Protective effects of delayed NAm treatment on behavioral defects in the EAE model. $A$, Behavioral scores (mean \pm SEM) of EAE in untreated $(n=14)$ or delayed treated (DT) $(n=12)$ groups. Differences between these groups were significant as determined by twotailed Student's $t$ test ( $p<0.01$ from $13 \mathrm{~d}$ p.i.). The differences seen before $10 \mathrm{~d}$ p.i. are attributable to the death of three mice in the untreated group when they showed clear behavioral deficits. $B$, NAD levels (mean \pm SEM) of cervical spinal cords from untreated or delayed treated groups. At both 2 and 4 weeks p.i., the NAD levels in the treated groups were significantly higher than those in untreated animals $\left({ }^{*} p<0.05 ;{ }^{* *} p<0.001\right.$; Student's $t$ test). The arrows indicate the starting point of delayed treatment in both $\boldsymbol{A}$ and $\boldsymbol{B}$. 
A

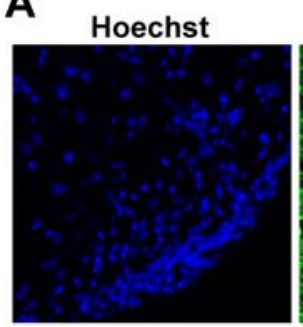

B

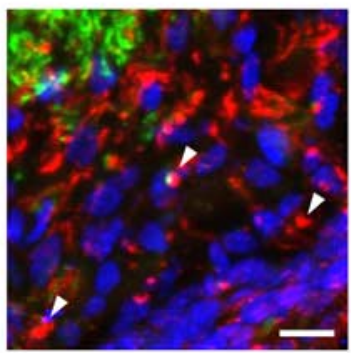

C
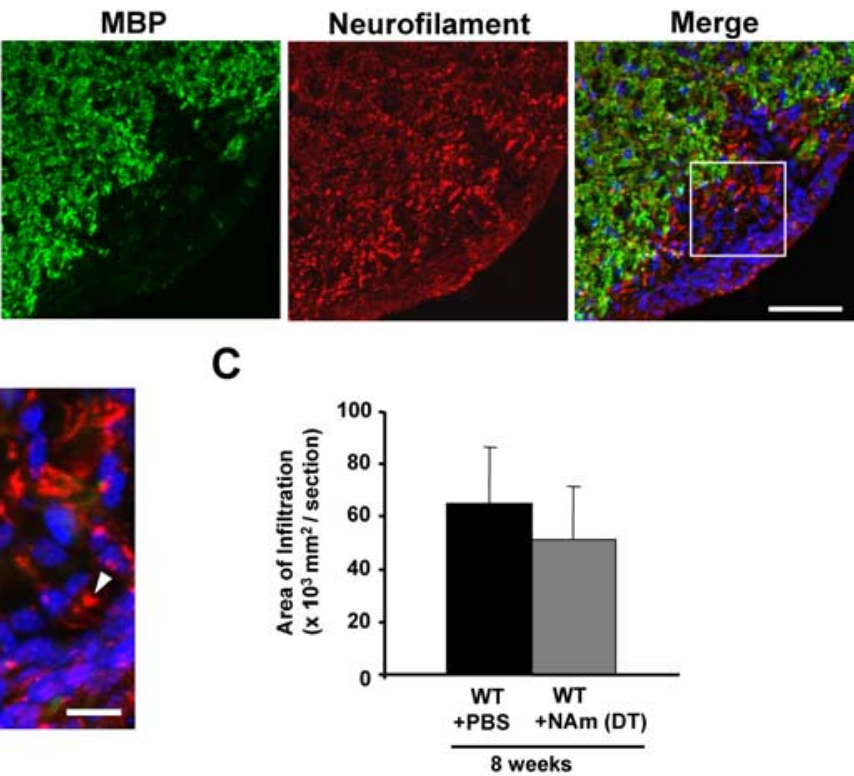

E

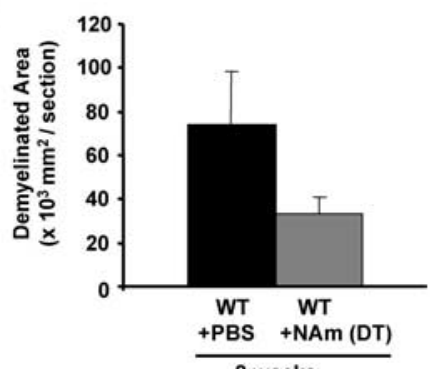

8 weeks

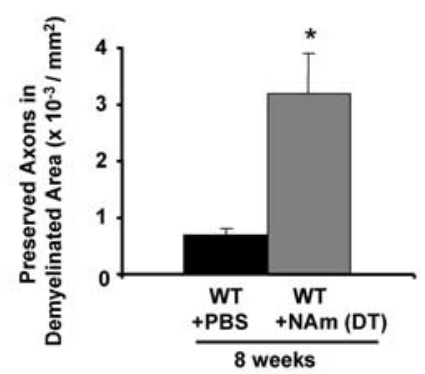

Figure 10. Protective effects of delayed NAm treatment on inflammation, demyelination, and axonal loss in the EAE model. $\boldsymbol{A}$, $\boldsymbol{B}$, Representative images showing the effects of delayed NAm treatment on infiltration, demyelination, and axonal loss. Transverse sections from wild-type EAE animals with delayed NAm treatment at 8 weeks p.i. were stained with Hoechst 33258 and antibodies against MBP and NF $(\boldsymbol{A})$. Higher magnification of the merged image is shown in $\boldsymbol{B}$. The arrowheads indicate several preserved axons in the demyelinated lesions. Scale bars: $\boldsymbol{A}, 50 \mu \mathrm{m} ; \boldsymbol{B}, 10 \mu \mathrm{m}$. $\boldsymbol{C}-\boldsymbol{E}$, Quantification of the average areas of infiltration $(p=0.65)(C)$ or demyelination $(p=0.14)(D)$ showed no significant difference, but the average number of $\mathrm{NF}+/ \mathrm{MBP}-$ fibers in demyelinated areas was increased in animals with delayed treatment with NAm ${ }^{*} p<0.05$; Student's $t$ test) (E). Error bars indicate SEM. 